\title{
STABILITY THEOREMS FOR WEDGES
}

\author{
by G. BROWN and J. D. PRYCE \\ (Received 21st November 1969)
}

\section{Introduction}

We are concerned with the stability properties of uniformly closed wedges in $C(E)$ (resp. $C^{+}(E)$ ), the real-valued (resp. non-negative) continuous functions on a compact space $E$, and solve the following problems in this area:

(a) Let $A$ be a closed semi-algebra in $C^{+}(E)$ such that

$$
f, g \in A \Rightarrow f g /(1+g) \in A \text {. }
$$

Is $A$ an ideal of a type 1 semi-algebra?

(b) Let $W$ be a closed wedge in $C^{+}(E)$ stable under some continuous $F: R^{+} \rightarrow R^{+}$with $\lim _{x \rightarrow \infty}(F(x)-x+1)=0$. Is $W$ stable under all increasing convex $G: R^{+} \rightarrow R^{+}$with $G(0)=0$ ?

(c) Let $n$ be a positive integer and let $A_{n}$ denote the semi-algebra of vectors in $R^{n+1}$ whose first $n$ differences are non-negative. Is $A_{n}$ stable under all $F: \boldsymbol{R}^{+} \rightarrow \boldsymbol{R}^{+}$whose first $n$ differences are non-negative?

Here, $W$ is said to be stable under $F$ if

$$
f \in W \Rightarrow F \circ f \in W \text {. }
$$

We give affirmative answers to $(a),(b),(c)$ in $\S \S 2,3,4$ respectively. Question (c) has irked us for some time-chiefly because it is tempting to guess that $A_{n}$ is the restriction to $\{i / n: 0 \leqq i \leqq n\}$ of the corresponding semi-algebras on $[0,1]$ (which motivated early semi-algebra theory). But this is false: in fact, already for $n=3$ the vector $(0,0,1,3)$ cannot be obtained in this way. A special case of $(b)$ under the additional hypothesis that $F$ is increasing and convex was proved by the second author in (9), and the " convexity " assumption was removed by F. F. Bonsall in (5) where the problem is raised in its present form and a positive answer conjectured. Problem (a) is already implicit in (4) and was considered in (1). Our solution is obtained as a consequence of a general stability theorem which has other interesting implications which we discuss also in $\$ 2$ (Theorems 3 and 4).

We wish to thank Professor F. F. Bonsall for a pre-publication copy of (5), and Professor E. J. Barbeau for finding a slip in the statement of Theorem 1.

\section{A general stability theorem}

Before stating the main theorem of this section (Theorem 1) we give a general condition for the existence of a suitable "choice" function to be used in the proof. 
Let $\mathscr{F}$ denote the class of all continuous functions $F: R^{+} \rightarrow R^{+}$which are increasing, convex, and satisfy $F(0)=0$.

Lemma 1. Let $F \in \mathscr{F}$ be non-linear and let $W$ be a closed wedge in $C^{+}(E)$ stable under $F$. Then there exists a sequence $\left(F_{n}\right)$ of functions in $\mathscr{F}$ such that

(i) $W$ is stable under $F_{n}, n=1,2, \ldots$,

(ii) $F_{n}(x) \rightarrow 0(x<1), F_{n}(x) \rightarrow \infty(x>1)$.

Proof. Let $\mathscr{S}(W)$ denote the set of functions in $\mathscr{F}$ under which $W$ is stable. $\mathscr{S}(W)$ is clearly composition closed and stable under multiplication by positive constants.

By the definition of convexity,

$$
F(t a)=F(t a+(1-t) 0) \leqq t F(a)+(1-t) F(0)=t F(a) \quad\left(a \in R^{+}, 0<t<1\right) .
$$

Since $F$ is non-linear there exist $a \in R^{+}$and $t$ with $0<t<1$ such that $F(t a)<t F(a)$. In particular $F(a)=b>0$ and we define $G \in \mathscr{S}(W)$ by

$$
G(x)=b^{-1} F(x a) \quad\left(x \in R^{+}\right) .
$$

Since $G(1)=1, G(t)<t$ we have

$$
G(x)<x \quad(0<x<1), \quad G(x)>x \quad(x>1) .
$$

In fact, with $\alpha=x / t, \beta=(x-t) /(1-t), \gamma=\beta^{-1}$, we have

$$
\begin{array}{ll}
G(x)=G(\alpha t+(1-\alpha) 0) \leqq \alpha G(t)+(1-\alpha) G(0)<x & (0<x<t), \\
G(x) \leqq \beta G(1)+(1-\beta) G(t)<x & (t<x<1), \\
G(x) \geqq \gamma^{-1}(G(1)-(1-\gamma) G(t))>x & (x>1) .
\end{array}
$$

We define $F_{n} \in \mathscr{S}(W)$ by

$$
F_{1}=G, F_{n}=G \circ F_{n-1} \quad(n=2,3 \ldots) .
$$

For $x<1$, the bounded sequence $\left(F_{n}(x)\right)$ is monotonic decreasing to a fix-point of $G$ and hence converges to zero. For $x>1$, the monotonic increasing sequence $\left(F_{n}(x)\right)$ must be unbounded for otherwise it would converge to a fix-point of $G$ greater than 1 . As each $F_{n}$ belongs to $\mathscr{S}(W)$ this completes the proof.

Notation. Let $W$ be a closed wedge in $C^{+}(E)$. We write

$$
[W]=\left\{f \in C^{+}(E): f g \in W \text { whenever } g \in W\right\} \text {. }
$$

Note that $[W]$ is a closed semi-algebra which contains the unit function, that $W \subseteq[W]$ if and only if $W$ is a semi-algebra, and that $W$ is then an ideal of $[W]$.

Theorem 1. Let $W$ be a closed wedge in $C^{+}(E)$ stable under a continuous function $G:\left(\boldsymbol{R}^{+}\right)^{2} \rightarrow \boldsymbol{R}^{+}$such that, for each $y, G(0, y)=0$ and

$$
\lim _{x \rightarrow \infty, y^{\prime} \rightarrow y} G\left(x, y^{\prime}\right)=y \text {. }
$$

Then $[W]$ is stable under all continuous increasing $H: R^{+} \rightarrow R^{+}$. In particular $[W]$ is a type 1 semi-algebra. 
If, in addition, $W$ is stable under some non-linear $F \in \mathscr{F}$ then $W$ is an ideal of the semi-algebra $[W]$ and, in particular, $W$ is stable under all elements of $\mathscr{F}$.

Corollary. The closed ideals of the type 1 semi-algebras in $C^{+}(E)$ are precisely those closed semi-algebras $A$ in $C^{+}(E)$ which have the following property:

$$
f, g \in A \Rightarrow f g(1+g)^{-1} \in A \text {. }
$$

Remark. E. J. Barbeau showed in (1) that a closed semi-algebra in $C^{+}(E)$ which is an inf-wedge is an ideal of a type 1 semi-algebra-a fact we can deduce from Theorem 1 by taking $G(x, y)=x \wedge y$ and $F(x)=x^{2}$ (since a semi-algebra admits squaring). On the other hand there exist ideals of type 1 semi-algebras which are not inf-wedges-see (4, p. 113). Thus, of the functions $x \wedge y$ and $x y /(1+y)$ on $\left(R^{+}\right)^{2}$, which both satisfy the conditions of Theorem 1 , stability under the first implies stability under the second but not vice-versa. There is scope here for study of two-dimensional stability properties in more generality.

Proof of theorem. Since $1 \in[W]$ we may as well consider continuous increasing $H: R^{+} \rightarrow R^{+}$with $H(0)=0$. Suppose that $f \in[W]$ but $H \circ f \notin[W]$ for some such $H$. Then there exists $g \in W$ such that $(H \circ f) g \notin W$. By the version of the Hahn-Banach theorem appropriate to ordered spaces there is a continuous linear functional $\mu$ on $C(E)$ such that

$$
\mu((H \circ f) g)=1, \quad \mu(h) \leqq 0 \quad(h \in W) .
$$

For $\alpha>0, n$ a positive integer we define $K_{\alpha, n}$ and $u_{\alpha}$ by

and

$$
K_{\alpha, n}=G \circ\left(\left(\alpha^{-1} f\right)^{n} g, g\right)
$$

$$
u_{\alpha}(x)= \begin{cases}1 & f(x)>\alpha \\ 0 & f(x) \leqq \alpha\end{cases}
$$

Because $|\mu|$ is a countably-additive measure there exists a countable set $\Gamma \subseteq R^{+}$such that $E_{\alpha}=\{x \in E: f(x)=\alpha\}$ is $|\mu|$-null for $\alpha \in R^{+} \sim \Gamma$, and it is easy to check that, outside $E_{\alpha}, K_{\alpha, n}$ converges pointwise to $g u_{\alpha}$ as $n \rightarrow \infty$. Since $G$ is bounded on $R^{+} \times$range $g$ it follows that $K_{\alpha, n}$ is bounded uniformly with respect to $n$, and an application of Lebesgue's dominated convergence theorem shows that

$$
\lim _{n \rightarrow \infty} \int K_{\alpha, n} d \mu=\int g u_{\alpha} d \mu \quad\left(\alpha \in R^{+} \sim \Gamma\right) .
$$

But $[W]$ is a semi-algebra so that $\left(\alpha^{-1} f\right)^{n} g \in W$ and hence $K_{\alpha, n} \in W$. In conjunction with (1) and (2) this proves that

$$
\int g u_{\alpha} d \mu \leqq 0 \quad\left(\alpha \in R^{+} \sim \Gamma\right) .
$$

Now fix $\varepsilon>0$ and choose $\alpha_{1}, \alpha_{2}, \ldots \alpha_{n} \in R^{+} \sim \Gamma, \alpha_{0}=0$, such that

$$
0<\alpha_{1}<\alpha_{2}<\ldots<\alpha_{n}, \max _{E} f \leqq \alpha_{n}, \quad H\left(\alpha_{k}\right)-H\left(\alpha_{k-1}\right)<\varepsilon, \quad k=1, \ldots, n .
$$


Defining $h=\sum_{k=1}^{n}\left(H\left(\alpha_{k}\right)-H\left(\alpha_{k-1}\right)\right) u_{\alpha_{k}} g$, we deduce from (3) that $\int h d \mu \leqq 0$, and a little calculation shows that $\left|\int((H \circ f) g-h) d \mu\right| \leqq \varepsilon \int g d|\mu|$. For small $\varepsilon$ these inequalities contradict (1) and establish the first part of the statement of the theorem. The particular choice $H(x)=x(1+x)^{-1}$ shows that $[W]$ is a type 1 semi-algebra.

Now suppose that $W$ is $F$-stable for some non-linear $F$ in $\mathscr{F}$. Let $\left(F_{n}\right)$ be a sequence of the kind guaranteed by Lemma 1 , and redefine $K_{\alpha, n}$ by

$$
K_{\alpha, n}=G \circ\left(F_{n} \circ\left(\alpha^{-1} f\right), g\right),
$$

where $g$ and $f$ are fixed elements of $W$. Repetition of the previous argument shows that $(H \circ f) g \in W$. Taking $H(x)=x$ we see $W$ is a semi-algebra and hence an ideal of $[W]$. The final assertion follows from the fact that every $F \in \mathscr{F}$ can be written as $x H(x)$ where $H$ is increasing on $R^{+}$. Thus

$$
f \in W \Rightarrow F \circ f=(H \circ f) f \in W .
$$

Remark. Our method of proof is based on the technique employed by F. F. Bonsall in obtaining Theorems 1,2 of (5). His results are stated for general topological spaces $E$ when $C(E)$ has the topology of uniform convergence on compacta-the extension of the preceding theorem to that case is straightforward. Theorem 2 of (5) is an easy corollary of our result and a similar extension of Theorem 1 of that paper is given below.

Theorem 2. Let $W$ be a closed wedge in $C^{+}(E)$ stable under a continuous function $G:\left(\boldsymbol{R}^{+}\right)^{2} \rightarrow \boldsymbol{R}^{+}$such that, for each $y, G(0, y)=y$ and

$$
\lim _{x \rightarrow \infty, y^{\prime} \rightarrow y} G\left(x, y^{\prime}\right)=0 .
$$

Then $[W]$ is stable under all continuous $H: R^{+} \rightarrow R^{+}$. In particular $[W]$ is a type 0 semi-algebra.

If, in addition, $W$ is stable under some non-linear $F \in \mathscr{F}$ then $W$ is an ideal of the semi-algebra $[W]$ and is itself stable under all continuous $H: \boldsymbol{R}^{+} \rightarrow \boldsymbol{R}^{+}$ with $H(0)=0$. Moreover $W-W$ is a closed subalgebra of $C(E)$ and

$$
W=(W-W) \cap C^{+}(E) .
$$

Corollary. The closed ideals of the type 0 semi-algebras in $C^{+}(E)$ coincide with the positive parts of the closed subalgebras of $C(E)$ and are precisely those closed semi-algebras in $C^{+}(E)$ which have the following property:

$$
f, g \in A \Rightarrow f(1+g)^{-1} \in A \text {. }
$$

Proof of theorem. A method similar to the previous proof shows first that $[W]$ is stable under decreasing continuous $H: \boldsymbol{R}^{+} \rightarrow \boldsymbol{R}^{+}$and hence (taking $H(x)=\frac{1}{1+x}$ ) is a type 0 semi-algebra. That the closed type 0 semialgebras are the positive parts of the closed unital subalgebras of $C(E)$ is the 
first result of semi-algebra theory (F. F. Bonsall (3)) and it follows that the word "decreasing" can be omitted. The proof that $W$ is an ideal if $F$-stable for non-linear $F$ in $\mathscr{F}$ is also similar to the previous proof and the remaining assertions follow easily from the fact that a closed ideal of a type 0 semi-algebra is the positive part of a closed subalgebra. This is a simple consequence of results in (4) but does not appear to have been explicitly stated. We give the following argument for completeness. Let $W$ be a closed ideal in a closed type 0 semi-algebra $A . W$ is obviously a type 1 semi-algebra and is thus of the form

$$
W=\left\{f \in C^{+}(E): f(x) \leqq f(y) \text { whenever } x \leqq_{W} y \text { and } f(x)=0 \quad(x \in N)\right\},
$$

where $\leqq_{W}$ is the partial order defined by

$$
x \leqq_{W} y \Leftrightarrow f(x) \leqq f(y) \quad(f \in W)
$$

and $N$ is the null set of $W$,

$$
N=\{x \in E: f(x)=0 \quad(f \in W)\} .
$$

We note also that the positive part of a closed subalgebra can be characterized in a similar fashion (via the Stone-Weierstrass theorem) where the partial order is, in fact, an equivalence relation. Accordingly it will suffice to prove that

$$
f \in W, 0<f(x)<f(y) \Rightarrow x{ }_{W} y .
$$

Given $f \in W, y \in E$, it follows that $f(y) 1-f \in A-A$, hence that

$$
(f(y) 1-f) \vee 0 \in A \text {, }
$$

and consequently $g=\left(f(y) f-f^{2}\right) \vee 0 \in W$. But if $0<f(x)<f(y)$, then

$$
g(x)>0=g(y) \text {. }
$$

This proves (1) and yields the required result.

Remarks. Theorem 2 is of less interest than Theorem 1, in the sense that whereas a two-dimensional stability function seems appropriate to characterize type 1 ideals among the wedges in $C^{+}(E)$, it is over-powerful for the type 0 ideals, where in fact a pair of one-dimensional functions will suffice. We recall from (6), Theorem 3 that a closed wedge $W$ in $C^{+}(E)$ is a type 1 semialgebra if and only if

$$
f \in W \Rightarrow f^{2} \in W \text { and } f-\frac{1}{2} f^{2} \in W \text { whenever }\|f\| \leqq 1 .
$$

This together with a slight extension of the argument at the end of the preceding proof shows that $W$ is an ideal of a type 0 semi-algebra if and only if

$$
f \in W \Rightarrow f^{2} \in W \text { and }\left(f-\frac{1}{2} f^{2}\right)^{+} \in W .
$$

(Of course the latter condition implies $\left(\lambda f-f^{2}\right)^{+} \in W$ for all $f \in W, \lambda \geqq 0$.) It is natural to ask whether the pair of functions $x^{2},\left(x-\frac{1}{2} x^{2}\right)^{+}$can be replaced by any non-linear $F \in \mathscr{F}$ together with any non-zero continuous $G: \boldsymbol{R}^{+} \rightarrow \boldsymbol{R}^{+}$ such that $G(0)=\lim _{x \rightarrow \infty} G(x)=0$. 
A summary of our knowledge of type 1 ideals in $C^{+}(E)$ may be in order. We have given an algebraic description of those wedges which can be ideals of a suitably chosen type 1 semi-algebra. E. J. Barbeau (1, p. 212) has characterized the class $\mathscr{J}_{A}$ of ideals of a given type 1 semi-algebra $A$ in $C^{+}(E)$ in terms of an elementary subclass $\mathscr{E}$ of $\mathscr{J}_{A}$ : a result " discovered" recently by the second author and at least one other worker in the field. Barbeau's result is as follows:

Let $\mathscr{U}$ denote the class of all closed upper sets in $E$ with respect to the order $\leqq_{A^{*}}$. For any $\mu \in C(E)^{*}$ define

$$
I_{\mu}=\left\{f \in A: \int_{U} f d \mu \geqq 0 \text { for all } U \in \mathscr{U}\right\} \text {. }
$$

Then $I_{\mu}$ is an ideal of $A$ and the set $\mathscr{E}$ of all such $I_{\mu}$ is an elementary class for $\mathscr{J}_{A}$.

In the special case where $E$ is a compact subset of $R$ with the usual ordering, A. K. Roy (10) has recently characterized those $\mu$ for which $I_{\mu}$ admits inf, sup, or both, and has shown that each ideal of one of these types is an intersection of $I_{\mu}$ of the same type; while B. A. Barnes (2) has found a simple description of those $\mu$ for which $J_{\mu}=\{f \in A: \mu(f) \geqq 0\}$ is an ideal of $A$ (which occurs if and only if $J_{\mu}$ and $I_{\mu}$ coincide).

Theorem 3. If $W$ is a closed inf-wedge in $C^{+}(E)$ then the following are equivalent:

(i) $W$ is stable under some non-linear $F \in \mathscr{F}$.

(ii) $W$ is stable under all $F \in \mathscr{F}$.

(iii) $W$ is a type 1 semi-algebra ideal.

The closed inf-wedges in $C^{+}(E)$ satisfying the equivalent conditions (i)-(iii) are the subsets $W$ of $C^{+}(E)$ of the form $W=\bigcap_{i \in I} A_{x_{i}, \mu_{i}}$ where for $x_{i} \in E, \mu_{i} a$ non-negative measure on $E$,

$$
A_{x_{i}, \mu_{i}}=\left\{f \in C^{+}(E): \int f d \mu_{i} \leqq f\left(x_{i}\right) \text { and } f(x) \leqq f\left(x_{i}\right) \quad\left(x \in \operatorname{supp} \mu_{i}\right)\right\} .
$$

Proof. A wedge $W$ is an inf-wedge if it is stable under $G(x, y)=x \wedge y$ so that the equivalence of (i), (ii), (iii) follows from Theorem 1 . It is elementary to verify that $\cap A_{x_{i}, \mu_{i}}$ is an inf-wedge satisfying (i). Let us suppose then that $W$ is a closed inf-wedge satisfying (ii). By the celebrated Choquet-Deny theorem (7),

$$
W=\bigcap_{i \in I} W_{x_{i}, \mu_{i}} \text { where } W_{x_{i}, \mu_{i}}=\left\{f \in C^{+}(E): \int f d \mu_{i} \leqq f\left(x_{i}\right)\right\} .
$$

If $\mu_{i}=0$ there is no difficulty. Suppose that for some $i$, we have

$$
f(x)>f\left(x_{i}\right) \text { for some } x \in \operatorname{supp}\left(\mu_{i}\right) \text {. }
$$


Then $\int\left(f-f\left(x_{i}\right)\right)_{+} d \mu_{i}>0=\left(f-f\left(x_{i}\right)\right)_{+}\left(x_{i}\right)$, and this leads to a contradiction since $W$ is stable under the function $F(x)=(x-\lambda)_{+}$, for constant $\lambda \geqq 0$.

Remark. The implication (i) $\Rightarrow$ (ii) under the above conditions with "concave" replacing " convex" is of long standing. (See (8), Theorem 1.)

In general the stability properties of wedges in $C(E)$ are more complicated than those of wedges of non-negative functions. It is of interest therefore to reduce the structure theory of inf-wedges in $C(E)$ stable under squaring to the case covered by Theorem 3. The obvious way to obtain a wedge of this kind is to take all elements of a closed subalgebra of $C(E)$ whose restrictions to some closed subset $F$ of $E$ form a square-stable inf-wedge in $C^{+}(F)$. In fact this simple process exhausts the possibilities.

Theorem 4. Let $W$ be a closed inf-wedge in $C(E)$ which is stable under squaring. Then there exists a closed subalgebra $A$ of $C(E)$, a closed subset $F$ of $E$, and a closed inf-wedge $W^{\prime}$ in $C^{+}(F)$ stable under squaring, such that

$$
W=\left\{f \in A:\left.f\right|_{F} \in W^{\prime}\right\},
$$

where $\left.f\right|_{F}$ is the restriction of $f$ to $F$. In particular $W$ is a semi-algebra.

Proof. Let $U=(-W) \cap C^{+}(E)$. Then from Theorem 4 of $(5)$ it follows that $U \subseteq W \cap(-W)$ and $U$ is the positive part of a closed subalgebra of $C(E)$. We show first that $W$ is a semi-algebra. Since $W$ is an inf-wedge,

$$
f \in W \Rightarrow-f_{-}=f \wedge 0 \in W .
$$

Hence for each $f$ in $W, f_{-} \in U \subseteq W \cap(-W)$. Also $f \vee 0=f_{+}=f+f_{-} \in W$. By Theorem $1, W \cap C^{+}(E)$ is a semi-algebra, so that

$$
f, g \in W \Rightarrow f_{+} g_{+}, f_{-} g_{-} \in W .
$$

Now fix $f, g \in W$. By square-stability, for each $\lambda>0$,

$$
\left(g_{-}-\lambda f_{+}\right)^{2}=g_{-}^{2}-2 \lambda f_{+} g_{-}+\lambda^{2} f_{+}^{2} \in W .
$$

Since $g_{-} \in U$, it follows that $g_{-}^{2} \in(U-U) \cap C^{+}(E)=U \subseteq-W$, so that

$$
-f_{+} g_{-}+\frac{1}{2} \lambda f_{+}^{2} \in W, \quad \lambda>0 .
$$

Letting $\lambda \rightarrow 0$, we deduce that $-f_{+} g_{-} \in W$, and similarly we obtain that $-g_{+} f_{-} \in W$.

It is now clear that $f g=\left(f_{+}-f_{-}\right)\left(g_{+}-g_{-}\right) \in W$, and we have proved that $W$ is a semi-algebra. by

Let $F=\{x \in E: f(x)=0(f \in U-U)\}$ and define the relation $\sim$ on $E \sim F$

$$
x \sim y \Leftrightarrow f(x)=f(y) \quad(f \in U-U) .
$$

Let $A$ be the closed subalgebra defined by

$$
A=\{f \in C(E): f(x)=f(y) \text { whenever } x \sim y\} .
$$


We now show that $W$ respects $\sim$. In fact, given $x, y \in E \sim F$ with $x \sim y$ there exists $h$ in $U-U$ such that $h \geqq 0$ and $h(x)=h(y)=1$. But

$$
U=(U-U) \cap C^{+}(E)
$$

so that $h \in U \subseteq-W$. Then, for each $f$ in $W, h f_{+}$and $h f_{-}$belong to

so that

$$
-W \cap C^{+}(E)=U \text {, }
$$

and hence

$$
h f=h f_{+}-h f_{-} \in U-U,
$$

$$
f(x)=h(x) f(x)=h(y) f(y)=f(y) .
$$

It is clear that the restriction to $F$ of each $f$ in $W$ is non-negative (since $f_{-} \in U$ whenever $f \in W$ ) and it follows immediately that these restrictions form a closed inf-wedge $W^{\prime}$ which is stable under squaring. Finally suppose that $g \in A, g \equiv f$ on $F$ for some $f$ in $W$. Then $g-f$ belongs to closed subalgebra $U-U$ (by the Stone-Weierstrass theorem) and, since $U-U \subseteq W$,

$$
g=f+(g-f) \in W .
$$

This completes the proof.

\section{Type 2 wedges}

In this section we answer question $(b)$ of the Introduction.

Lemma 2. Let $F: R^{+} \rightarrow R^{+}$be continuous with $\lim _{x \rightarrow \infty}(F(x)-x+1)=0$. Let $F_{1}=F$, and $F_{n}=F \circ F_{n-1}(n=2,3, \ldots)$. Then

uniformly on $\boldsymbol{R}^{+}$as $n \rightarrow \infty$.

$$
\frac{1}{n} F_{n}(n x) \rightarrow(x-1)^{+}
$$

Remark. This result under the additional assumption that $F$ be increasing is proved by Bonsall $(5$, p. 140$)$, and we make use of this result in the present proof.

Proof. Define the functions $U$ and $L$ by

$$
\begin{aligned}
& U(x)=\sup \{F(t): 0 \leqq t \leqq x\} \quad\left(x \in R^{+}\right) . \\
& L(x)=\inf \{F(t): x \leqq t<\infty\}
\end{aligned}
$$

Since $F$ is non-negative, and bounded on bounded intervals, $U$ and $L$ are finite, non-negative, continuous and increasing, and satisfy

$$
L(x) \leqq F(x) \leqq U(x) \quad\left(x \in R^{+}\right) .
$$

It follows at once from the definition of $L$ that if for any $\varepsilon>0$ we have

$$
|F(t)-t+1| \leqq \varepsilon
$$

for all $t \geqq x_{0}$, then $\left|L\left(x_{0}\right)-x_{0}+1\right| \leqq \varepsilon$; replacing $x_{0}$ by any $x \geqq x_{0}$ we have $|L(x)-x+1| \leqq \varepsilon\left(x \geqq x_{0}\right)$ and so

$$
\lim (L(x)-x+1)=0 .
$$


We now show that $\lim (U(x)-x+1)=0$. In fact, choose any $\varepsilon>0$. There is $\xi_{0} \in R^{+}$such that $|F(x)-x+1|<\varepsilon\left(x \geqq \xi_{0}\right)$. Let

$$
\xi=\max \left(\xi_{0}, U\left(\xi_{0}\right)+1+2 \varepsilon\right) .
$$

For any $x \geqq \xi$ we have $U(x) \geqq F(x)>x-1-\varepsilon \geqq \xi-1-\varepsilon$ so that

$$
U(x)>U\left(\xi_{0}\right)+\varepsilon .
$$

There is $t \leqq x$ such that $F(t)>U(x)-\varepsilon>U\left(\xi_{0}\right)$. Then $t>\xi_{0}$ by the definition of $U$, so that

We then deduce

$$
|F(t)-t+1|<\varepsilon \text {. }
$$

$$
x-1-\varepsilon<F(x)<U(x)<F(t)+\varepsilon<(t-1+\varepsilon)+\varepsilon \leqq x-1+2 \varepsilon .
$$

Hence $|U(x)-x+1|<2 \varepsilon(x \geqq \xi)$ and it follows that $\lim (U(x)-x+1)=0$.

Now let $U_{n}, L_{n}$ be the $n$-th iterates of $U$ and $L$ defined in the same way as the $F_{n}$. An inductive argument using the monotonicity of $U$ and $L$ shows

$$
L_{n}(x) \leqq F_{n}(x) \leqq U_{n}(x) \quad\left(x \in R^{+}, n=1,2, \ldots\right) .
$$

Now $U$ and $L$ satisfy the conditions of Bonsall's lemma, so that $n^{-1} L_{n}(n x)$, $n^{-1} U_{n}(n x) \rightarrow(x-1)^{+}$uniformly on $R^{+}$as $n \rightarrow \infty$, and the desired result follows at once from (3.1).

Remark. Bonsall's proof makes no use of the continuity of $F$, so that ours uses it only in order to ensure that $F$ is bounded on bounded intervals.

Theorem 5. Let $F: R^{+} \rightarrow R^{+}$be continuous with $\lim _{x \rightarrow \infty}(F(x)-x+1)=0$, and let $W$ be a closed wedge in $C^{+}(E)$ stable under $F$. Then $W$ is reductive, type 2 , and stable under all $G \in \mathscr{F}$.

Remark. Reductive and type 2 mean stable under the functions $(x-1)^{+}$, $x^{2} /(1+x)$ respectively.

Proof. Let $\left\{F_{n}\right\}$ be the sequence of the previous lemma. For any $f \in W$,

$$
\frac{1}{n} F_{n} \circ(n f) \in W \quad(n=1,2, \ldots) .
$$

As $n \rightarrow \infty$ this sequence converges uniformly to $(f-1)^{+}$and hence $W$ is reductive.

That a closed reductive wedge in $C^{+}(E)$ is stable under all elements of $\mathscr{F}$ is an early result of stability theory (see (9), (5)) and the type 2 property is a particular case of this.

\section{Semi-algebras of completely monotonic vectors}

Notation. Given a continuous function $F: I \rightarrow R$ where $I$ is an interval (possibly infinite) in $R$, and given $h \in R^{+}$, we define $\Delta_{h} F$ by

$$
\text { E.M.S. }-0
$$

$$
\Delta_{h} F(t)=F(t+h)-F(t)
$$


whenever $t, t+h \in I$. We write $\Delta_{h}^{2} F$ for $\Delta_{h}\left(\Delta_{h} F\right)$, etc., and we have the general formula for the $n$-th difference of $F$ (of length $h$ ):

$$
\Delta_{h}^{n} F(t)=\sum_{r=0}^{n}\left(\begin{array}{l}
n \\
r
\end{array}\right)(-1)^{n-r} F(t+r h)
$$

defined whenever $t,(t+h), \ldots,(t+n h) \in I$.

Definition. $F$ is called $n$-fold monotonic if $F \geqq 0$ and for $k=1,2, \ldots, n$ and each $h>0$ we have $\Delta_{h}^{k} F(t) \geqq 0$ whenever it is defined.

Notation. Let $J$ be an interval $\{i: p \leqq i \leqq q\}$ in $Z$ and let $x: J \rightarrow R$, so that $x$ may be thought of as a vector $\left(x_{p}, \ldots, x_{q}\right)$ in space of $q-p+1$ dimensions. Similarly to above we define the $n$-th difference of $x$ by

$$
\left(\Delta^{n} x\right)_{i}=\sum_{r=0}^{n}\left(\begin{array}{l}
n \\
r
\end{array}\right)(-1)^{n-r} x_{i+r}
$$

whenever $p \leqq i \leqq i+n \leqq q$. Clearly $\Delta^{n} x$ is a vector with $q-p-n+1$ components $(n \leqq q-p)$ but fails to be defined if $n>q-p$. We shorten $\left(\Delta^{n} x\right)_{i}$ to $\Delta^{n} x_{i}$.

Definition. $x$ is called completely monotonic (c.m.) if $\left(\Delta^{n} x\right)_{i} \geqq 0$ whenever it is defined.

Notation. We denote by $A_{n}(n=0,1,2, \ldots)$ the set of all $x:\{0,1, \ldots, n\} \rightarrow R$ which are c.m.; which we can regard as a subset of $\boldsymbol{R}^{n+1}$.

Clearly $A_{n}$ is a cone in $R^{n+1}$. With multiplication defined pointwise it is well known that

$$
A_{n} \text { is a semi-algebra. }
$$

Given continuous $F: R^{+} \rightarrow R^{+}$and $x \in A_{n}$ we denote by $F \circ x$ the function composition of $F$ and $x$, so that in the vector notation $F \circ x=\left(F\left(x_{0}\right), \ldots, F\left(x_{n}\right)\right)$.

Theorem 6. For $n=0,1,2, \ldots$ the semi-algebra $A_{n}$ is stable under all $F: \boldsymbol{R}^{+} \rightarrow \boldsymbol{R}^{+}$which are continuous and $n$-fold monotonic.

Corollary. Since the function $t \rightarrow t^{n} /(1+t)$ is $n$-fold monotonic it follows that $A_{n}$ is a type $n$ semi-algebra.

Remark. The continuous analogue of this, namely that the semi-algebra $B_{n}$ of $n$-fold monotonic functions in $C[0,1]$ is stable under all continuous, $n$-fold monotonic $F: R^{+} \rightarrow R^{+}$, is of long standing and easily proved by differentiation, using induction and Lemma 3.

Preliminaries to the proof. We shall proceed by induction on $n$. The next lemma, a well-known result in the theory of convex functions, is used in reducing the problem to one with a smaller value of $n$.

Lemma 3. Let $F$ be as in the statement of the theorem. Then for $n \geqq 2, F$ is absolutely continuous and $F^{\prime}$ (suitable values being inserted at the points of a 
null set $)$ is $(n-1)$-fold monotonic. For $n \geqq 3$ we have the stronger result that $F$ is continuously differentiable on $\boldsymbol{R}^{+}$.

For a fixed $n$, let $u_{k}(0 \leqq k \leqq n)$ denote the vector

$$
\left(\left(\begin{array}{l}
0 \\
k
\end{array}\right),\left(\begin{array}{l}
1 \\
k
\end{array}\right), \ldots,\left(\begin{array}{l}
n \\
k
\end{array}\right)\right)
$$

Lemma 4. Each $x \in R^{n+1}$ is uniquely expressible as $\Sigma \lambda_{k} u_{k}$ where $\lambda_{k}=\Delta_{k} x_{0}$. In particular $A_{n}$ is precisely the conical hull of the $u_{k}$.

Proof. Let $S: \boldsymbol{R}^{n+1} \rightarrow \boldsymbol{R}^{n}, \boldsymbol{R}: \boldsymbol{R}^{n+1} \rightarrow \boldsymbol{R}^{n}$ be the operators defined by

$S\left(x_{0}, x_{1}, \ldots, x_{n}\right)=\left(x_{1}, x_{2}, \ldots, x_{n}\right), \quad R\left(x_{0}, x_{1}, \ldots, x_{n}\right)=\left(x_{0}, x_{1}, \ldots, x_{n-1}\right)$.

Since $R, S$ commute and $\Delta=S-R$ we have

But for, $0 \leqq k \leqq r$,

$$
S^{r}=(R+\Delta)^{r}=\sum_{k=0}^{r}\left(\begin{array}{l}
r \\
k
\end{array}\right) \Delta^{k} R^{r-k}
$$

so that

$$
\left(R^{r-k} \Delta^{k} x\right)_{0}=\left(\Delta^{k} x\right)_{0}
$$

$$
x_{r}=\left(S^{r} x\right)_{0}=\sum_{k=0}^{r}\left(\begin{array}{l}
r \\
k
\end{array}\right) \lambda_{k} \text { where } \lambda_{k}=\Delta^{k} x_{0}
$$

Since $\left(\begin{array}{l}r \\ k\end{array}\right)=0$ for $r+1 \leqq k \leqq n$ we may replace $\sum_{0}^{r}$ by $\sum_{0}^{n}$, thus

so that

$$
x_{r}=\sum_{k=0}^{n} \lambda_{k}\left(\begin{array}{l}
r \\
k
\end{array}\right) \quad(0 \leqq r \leqq n)
$$

$$
x=\sum_{0}^{n} \lambda_{k} u_{k}
$$

The number of $u_{k}$ is equal to the dimension of the space, so they form a basis and the expansion is unique. Hence, if $y=\Sigma u_{k} \mu_{k}$ then $\mu_{k}=\Delta^{k} y_{0}$.

It follows that each $y \in A_{n}$ is a conical combination of the $u_{k}$. Conversely, let $x=\Sigma \mu_{k} u_{k}$ with $\mu_{k} \geqq 0$. We need to show that $\Delta^{q} x_{r} \geqq 0(0 \leqq q \leqq q+r \leqq n)$, and this is true, since

$$
\begin{aligned}
\Delta^{q} x_{r} & =\Delta^{q} S^{r} x_{0}=\Delta^{q}(R+\Delta)^{r} x_{0} \\
& =\sum_{k=0}^{r}\left(\begin{array}{l}
r \\
k
\end{array}\right) \Delta^{q+k} x_{0}=\sum_{k=0}^{r}\left(\begin{array}{l}
r \\
k
\end{array}\right) \mu_{q+k} \geqq 0 .
\end{aligned}
$$

This completes the proof.

Proof of Theorem 6. Let $P(n)$ denote the assertion of the theorem. Assume inductively that $P(m)$ holds for $0 \leqq m<n$, where $n \geqq 2$. (It is:"easy to verify that $P(0)$ and $P(1)$ are satisfied.)

Let a fixed $n$-fold monotonic function $F$ be chosen. 
Notation. By a part of a vector $\left(x_{p}, \ldots, x_{q}\right)$ we shall mean a "subvector" of the form $\left(x_{p^{\prime}}, \ldots, x_{q^{\prime}}\right)$ where $p \leqq p^{\prime} \leqq q^{\prime} \leqq q$. It is clear from the definition that any part of a c.m. vector is c.m. From this fact and the fact that $F$ is certainly $k$-fold monotonic if $0 \leqq k<n$, we deduce by the inductive hypothesis that for

$$
x \in A_{n}, \quad \Delta^{k}(F \circ x)_{i} \geqq 0 \quad(0 \leqq k<n, 0 \leqq i \leqq i+k \leqq n)
$$

so that it only remains to prove that for any $x \in A_{n}$.

$$
\Delta^{n}(F \circ x)_{0} \geqq \geqq 0,
$$

this being the only $n$-th difference that exists.

By Lemma 4, this is equivalent to proving the non-negativity on the positive cone in $R^{n+1}$ of the function $f(\lambda)=\hat{F}\left(\lambda_{0}, \lambda_{1}, \ldots, \lambda_{n}\right)=\Delta^{n}(F \circ x)_{0}$ where $x=\Sigma \lambda_{k} u_{k}$. From the definitions,

$$
\hat{F}(\lambda)=\sum_{r=0}^{n}\left(\begin{array}{l}
n \\
r
\end{array}\right)(-1)^{n-r} F\left(\sum_{k=0}^{r} \lambda_{k}\left(\begin{array}{l}
r \\
k
\end{array}\right)\right)
$$

By Lemma 3, $F$ is absolutely continuous, so that $\hat{F}$ is also absolutely continuous with respect to each $\lambda_{k}$. Now,

$$
\begin{aligned}
\frac{\partial \hat{F}}{\partial \lambda_{k}} & =\sum_{r=0}^{n}\left(\begin{array}{l}
n \\
r
\end{array}\right)(-1)^{n-r}\left(\begin{array}{l}
r \\
k
\end{array}\right) F^{\prime}\left(\sum_{k=0}^{r} \lambda_{k}\left(\begin{array}{l}
r \\
k
\end{array}\right)\right) \\
& =\left(\begin{array}{l}
n \\
k
\end{array}\right) \sum_{r=0}^{n}(-1)^{n-r}\left(\begin{array}{l}
n-k \\
r-k
\end{array}\right)\left(F^{\prime} \circ x\right)_{r}, \quad\left(\operatorname{since}\left(\begin{array}{l}
n \\
r
\end{array}\right)\left(\begin{array}{l}
r \\
k
\end{array}\right)=\left(\begin{array}{l}
n \\
k
\end{array}\right)\left(\begin{array}{l}
n-k \\
r-k
\end{array}\right)\right) .
\end{aligned}
$$

Setting $s=r-k$ and discarding terms for which the inner binomial coefficient vanishes we obtain

$$
\begin{aligned}
& \frac{\partial \hat{F}}{\partial \lambda_{k}}=\left(\begin{array}{l}
n \\
k
\end{array}\right) \sum_{s=0}^{n-k}(-1)^{n-k-s}\left(\begin{array}{c}
n-k \\
s
\end{array}\right) y_{s}, \\
& \frac{\partial \hat{F}}{\partial y_{k}}=\left(\begin{array}{l}
n \\
k
\end{array}\right) \Delta^{n-k} y_{0},
\end{aligned}
$$

where $y=F^{\prime} \circ\left(x_{k}, x_{k+1}, \ldots, x_{n}\right)$. Now $\left(x_{k}, \ldots, x_{n}\right)$ is a part of the c.m. vector $x$, and hence c.m., and provided $k \geqq 1$ it follows by the inductive hypothesis and Lemma 3 that $y$ is c.m., whence

$$
\frac{\partial \hat{F}}{\partial \lambda_{k}} \geqq 0 \text { on }\left(R^{n+1}\right)^{+} \text {for } k=1, \ldots, n .
$$

By absolute continuity we thus have for any $\left(\lambda_{0}, \ldots, \lambda_{n}\right)$,

$$
\begin{aligned}
\hat{F}\left(\lambda_{0}, \ldots, \lambda_{n}\right) & \geqq \hat{F}\left(\lambda_{0}, 0, \ldots, 0\right) \\
& =\Delta^{n}\left(F \circ \lambda_{0} u_{0}\right)_{0} \\
& =\Delta^{n}\left(F\left(\lambda_{0}\right) .(1,1, \ldots, 1)\right)_{0}=0 .
\end{aligned}
$$

This establishes the induction step and the theorem is proved. 
Remarks. (i) It is easy to extend this result to deal with the semi-algebra of " $n$-fold monotonic" vectors in $R^{m+1}$ with $m>n$, that is, the set

$$
\begin{gathered}
A_{m, n}=\left\{x=\left(x_{0}, \ldots, x_{m}\right): \Delta^{k} x_{i} \geqq 0 \text { for } 0 \leqq k \leqq n, \text { and all } i\right. \\
\text { such that this is defined }\} .
\end{gathered}
$$

For clearly $x \in A_{m, n}$ if and only if each of its parts, of "length" $n+1$, is c.m. We thus obtain

$$
F n \text {-fold monotonic, } x \in A_{m, n} \Rightarrow F \circ x \in A_{m, n} \text {. }
$$

(ii) The case where $F$ is defined on an open or closed bounded subinterval of $R^{+}$can be dealt with in much the same way but with more technical detail since $F$ will generally not be extendable to an $n$-fold monotonic function on $R^{+}$, and $F^{\prime}$ may be unbounded even when $F$ is bounded.

\section{A problem on the representation of certain finite measures}

Let $X$ denote the space of continuous real functions on $R^{+}$with the topology of pointwise convergence. Then $X^{*}$, which we endow with the $w^{*}$-topology, is the space of all measures of finite support on $R^{+}$. The set

$$
\mathscr{F}_{n}=\{f \in X: f \text { is } n \text {-fold monotonic }\}
$$

is a closed wedge in $X$, being determined by the inequalities

$$
\mu_{h, t, k}(f)=\left(\sum_{r=0}^{k}\left(\begin{array}{l}
k \\
r
\end{array}\right)(-1)^{k-r} \varepsilon_{t+r h}\right)(f) \geqq 0 \quad\left(0 \leqq k \leqq n ; h, t \in R^{+}\right) .
$$

It is well known that the dual cone

$$
\mathscr{F}_{n}^{d}=\left\{\mu \in X^{*}: \mu(f) \geqq 0 \text { for all } f \in \mathscr{F}_{n}\right\}
$$

is therefore the closed conical hull of the measures $\mu_{h, t, k}$. Now Theorem 6 states that if $0 \leqq k \leqq n, x \in A_{k}$, and $v_{x}$ denotes the measure

$$
\left(\begin{array}{l}
k \\
0
\end{array}\right) \varepsilon_{x_{k}}-\left(\begin{array}{c}
k \\
1
\end{array}\right) \varepsilon_{x_{k-1}}+\ldots+(-1)^{k}\left(\begin{array}{l}
k \\
k
\end{array}\right) \varepsilon_{x_{0}}
$$

then $v_{x}(f)=\Delta^{k}(f \circ x)_{0} \geqq 0$ for all $f \in \mathscr{F}_{n}$; that is

$$
v_{x} \in \mathscr{F}_{n}^{d} \quad\left(x \in A_{k}\right)
$$

Hence each such $v_{x}$ is the limit of finite positive combinations of the $\mu_{h, t, k}$. Note that $\mu_{h, t, k}$ is a particular case of a $v_{x}$, in which

$$
x=(t, t+h, \ldots, t+k h) \in A_{k} .
$$

Question. Is it true that $v_{x}$ is in fact a finite positive combination of measures $\mu_{h, t, k}$ without the intervention of a limit process? If not, is there a somewhat extended representation possible, say one corresponding not to powers $\Delta_{h}^{k}$ but to products of different $\Delta_{h}$ leading to measures of the form

$$
\mu(f)=\left(\prod_{i=1}^{k} \Delta_{h_{i}}\right) f(t)
$$


It is easy to verify a formula of the above form in case $n=2$, and possible but tedious for $n=3$. The representation is not unique in either case, and it would be of interest to find a canonical form and an algorithm for obtaining this.

Example. In the case $n=2$, let us represent an arbitrary

$$
x=\left(x_{0}, x_{1}, x_{2}\right) \in A_{2}
$$

in the form $\lambda_{0} u_{0}+\lambda_{1} u_{1}+\lambda_{2} u_{2}$ according to Lemma 4 , where

Then

$$
u_{0}=(1,1,1), \quad u_{1}=(0,1,2), \quad u_{2}=(0,0,1)
$$

so that

$$
\begin{aligned}
v_{x}(f) & =f\left(x_{2}\right)-2 f\left(x_{1}\right)+f\left(x_{0}\right) \\
& =f\left(\lambda_{2}+2 \lambda_{1}+\lambda_{0}\right)-2 f\left(\lambda_{1}+\lambda_{0}\right)+f\left(\lambda_{0}\right) \\
& =\Delta_{\lambda_{2}} f\left(2 \lambda_{1}+\lambda_{0}\right)+\Delta_{\lambda_{1}}^{2} f\left(\lambda_{0}\right)
\end{aligned}
$$

$$
v_{x}=\mu_{\lambda_{2}, 2 \lambda_{1}+\lambda_{0,1}}+\mu_{\lambda_{1}, \lambda_{0}, 2}
$$

\section{REFERENCES}

(1) E. J. Barbeau, Commutative semi-algebras (Ph.D. Thesis, University of Newcastle upon Tyne, 1964).

(2) B. A. Barnes, (to appear).

(3) F. F. Bonsall, Semi-algebras of continuous functions, Proc. London Math. Soc. (3) 10 (1960), 1-16.

(4) F. F. Bonsall, Semi-algebras of continuous functions, Proc. Int. Symposium on Linear Spaces (Jerusalem 1960), 101-114.

(5) F. F. Bonsall, Stability theorems for wedges and semi-algebras, J. Functional Analysis 4 (1969), 135-145.

(6) G. Brown, Stability of wedges and semi-algebras, Proc. Cambridge Philos. Soc. 64 (1968), 365-376.

(7) G. Choquet and J. DenY, Ensembles semi-réticulés et ensembles réticulés de fonctions continues, J. Math. Pures Appl. 36 (1957), 179-189.

(8) S. GuBER, Über die Struktur gewisser Funktionenkegel, (Ph.D. Dissertation, Friedrich-Alexander-Universität Erlangen, 1961).

(9) J. D. Pryce, On type F semi-algebras of continuous functions, Quart. J. Math. Oxford (2) 16 (1965), 65-71.

(10) A. K. RoY, (to appear).

UNIVERSITY OF LIVERPOOL

UNIVERSITY OF ABERDEEN 